\title{
Universal Hinges and the Bounds of Sense
}

Mark Fortney

Forthcoming in Philosophia, 2019

7000 words

This is a penultimate draft. Please cite the published version at Philosophia's website.

\begin{abstract}
:
According to Danièle Moyal-Sharrock, Wittgenstein's On Certainty presents a theory of hinges, and hinges have a role to play in a foundationalist epistemology (2013, this journal). Michael Williams (2005) and Annalisa Coliva (2013, this journal) have claimed that the hinges are not suitable to play such a role as they are not shared universally. Moyal-Sharrock has replied that a subset of the hinges is suitable to play such a role: the "universal" hinges, an account of which she developed in her 2004 book Understanding on Certainty (2013, this journal). I argue that for Moyal-Sharrock's reply to be sustained, she must construe the set of universal hinges much more narrowly than she does currently. For instance, Moyal-Sharrock claims that "I have a brain" is a universal hinge, which consigns people who know nothing about brains to stand outside the bounds of sense. I also provide a novel way of thinking about the universal hinges, which I argue is better textually motivated than Moyal-Sharrock's own way, and which provides a set of hinges more suitable to play a role in foundationalist epistemology.
\end{abstract}




\section{Introduction}

According to Danièle Moyal-Sharrock, Wittgenstein's On Certainty presents a theory of hinges. On Moyal-Sharrock's view, hinges are "animal" and "nonepistemic" certainties of ours which partly constitute our framework for making sense of the world, and having this framework is what gives us the the capacity to engage in belief formation, maintenance, and revision. ${ }^{1}$ What makes hinges animal and nonepistemic for Moyal-Sharrock is that they are not subject to the kind of epistemic evaluations that we use to categorize beliefs.

Moyal-Sharrock argues additionally that hinges have an important role to play in a foundationalist epistemology, despite the fact that hinges are not beliefs. More generally, on her way of thinking, hinges underwrite our capacity to act and to participate in various forms of life. Because hinges have so many roles to play they are essential to understanding Wittgenstein's final account of epistemology, and this paper aims to advance our understanding of hinges. ${ }^{2}$

This paper is about the distinction between two of categories of hinges ("local" and "universal"), as well as the broader import that the universal hinges in particular have for questions about the nature of rationality and the foundations of our beliefs. I present a new way of understanding the universal hinges, and explain the significance this new reading has for both readers of Wittgenstein and philosophers interested in foundationalist epistemologies.

The distinction between local and universal hinges was introduced by MoyalSharrock in Understanding On Certainty. Local hinges are hinges shared by groups of people in a particular place and time, whereas universal hinges are shared by everyone within the "bounds of sense". ${ }^{3}$ The correct characterisation of the universal hinges is therefore of particular philosophical significance. After all, the way we answer the question "What is it

\footnotetext{
${ }^{1}$ Understanding On Certainty p. 67, p. 108. More generally, see "Ch 4: The Features of Hinges".

2 “On Coliva's Judgmental Hinges" p. 24

${ }^{3}$ Understanding On Certainty p. 103
} 
to make sense or be rational?" is going to be fixed by how we characterize the universal hinges, and is in itself a question of general philosophical significance.

I'll argue that Moyal-Sharrock frequently incorrectly categorizes local hinges as universal hinges, which results in developing an overly narrow way of construing the bounds of sense. Moreover, I'll argue that there is textual evidence that Wittgenstein would have agreed with my more inclusive way of thinking about the bounds of sense.

Additionally, while Moyal-Sharrock identifies universal hinges one by one, I move forward on the question of how to characterize universal hinges by specifying the underlying principle that explains why the universal hinges are universal.

On my interpretation, the genuinely Wittgensteinian universal hinges are those that are required to participate in language games, and therefore (as I will argue) the set of universal hinges includes hinges like "I am not the only person that exists" and "There is an external world". Moyal-Sharrock's account of universal hinges, by contrast, includes details about human life that go above and beyond this sparse account of the universal hinges. For instance, she counts "I have a brain" as one of the universal hinges. But one can obviously fail to know biological trivia like that and still manage to stand within the bounds of sense.

Lastly, I'll finish the paper by explaining how my account of the universal hinges turns out to be essential to Moyal-Sharrock's ambition of using hinges to play a role in a foundationalist epistemology. My account of the universal hinges is therefore motivated in three separate ways: it makes the bounds of sense appropriately inclusive, it's textually motivated by claims in On Certainty, and it suitable for playing the epistemological role that Moyal-Sharrock wants an account of universal hinges to be able to play.

\section{§1. Moyal-Sharrock on Local and Universal Hinges}

For Moyal-Sharrock, the set of local hinges varies with time, place, and culture. ${ }^{4}$ For example, consider the hinge "People can walk on the moon", which is currently a local hinge for me and many of the people that live in the same city that I do (problem all of them, but philosophers are cautious). It is a hinge that we acquire through training -

${ }^{4}$ Understanding On Certainty p. 102, p. 136 
continual repetition of facts that presuppose its truth might partly constitute such training. As a member of my culture, I have been frequently exposed to messages that took it for granted that people can walk on the moon, starting from fairly early on in my life, and this is why "People can walk on the moon" is a hinge for me.

By contrast, someone like Descartes was never exposed to (most of) the media and other background conditions that I was exposed to while acquiring most of my hinges, and for him "People can walk on the moon" was not a hinge. This is why "People can walk on the moon" is a local hinge: it is shared by some groups at some times, but not by all of them. As a result, Descartes and I had different frameworks for approaching the world and forming, maintaining, and revising beliefs about the world.

But on Moyal-Sharrock's way of thinking, there is at least some sort of overlap between the framework with which I approach the world and the framework with which Descartes approached the world. And that seems like it must be right. On her way of thinking, both of our sets of hinges must have included the universal hinges. According to Moyal-Sharrock, the universal hinges form the bounds of sense. They are, on her account, the minimal context that all forms of life have in common. ${ }^{5}$ Moyal-Sharrock also frames her account of universal hinges by contrasting Wittgenstein (with whom she agrees) with Quine (with whom she disagrees). While Quine would say that any commitment of ours can be given up, in the right sort of circumstance, Moyal-Sharrock argues that on Wittgenstein's view universal hinges (as well as some other hinges) just cannot be given up. ${ }^{6}$

Moyal-Sharrock argues that we can see evidence for this attitude in Wittgenstein's riverbank metaphor. Here is one of his descriptions of the riverbank metaphor (from $\mathrm{On}$ Certainty): "And the bank of the river consists partly of hard rock, subject to no alteration or only an imperceptible one". ${ }^{7}$ Moyal-Sharrock argues that it would be a mistake to think that any part of the hard rock of the riverbank (which corresponds to all of a person's hinges) is subject to only imperceptible alterations. Instead, she wants to read the passage as saying that some parts of the riverbank are subject to no alteration and some other parts of the

${ }^{5}$ Understanding On Certainty p. 150

6 Understanding On Certainty p. 103

${ }^{7}$ On Certainty $\$ 99$ 
riverbank are subject to imperceptible alterations. ${ }^{8}$ Thus, Moyal-Sharrock's claim is that what she identifies as universal hinges are what Wittgenstein would have called parts of the bedrock (i.e., the set of a person's hinges) that are not subject to any alteration.

With these general points about universal hinges on hand, we can move on to discussing some of Moyal-Sharrock's specific claims about of what the universal hinges are.

For example, Moyal-Sharrock suggests that "the world exists" is a universal hinge. ${ }^{9}$ The claim that such a hinge cannot be given up does seem reasonable. Our possession of hinges is shown in the way we live our lives, and it does not seem like a human could really deny - in their actions - that there is an external world while engaging in all sorts of typically human activities - talking, eating, walking. Of course, in the philosophy classroom someone might verbally express doubt about the external world, or even claim not to believe in its existence, but it seems like such a person is in fact just incorrectly characterizing what is a hinge that cannot be given up for them. And it is by now a familiar point that there is in many cases a vast gulf between what agents say that believe and what agents actually believe, and it seems reasonable to extrapolate that introspection is as liable to lead us astray about hinges as it is to lead us astray about beliefs. ${ }^{10}$

Moyal-Sharrock then goes on, to tell us that several other hinges are universal hinges, including Moore's truisms from his defense of common sense. ${ }^{11}$ I think a case similar to the one I made above could be made for each of, or at least the majority of, these hinges. These are hinges that seem like they cannot be genuinely given up by people acting in the world, despite the fact that people might claim to have beliefs or doubts that are incompatible with genuine acceptance of these hinges.

In the next section of the paper, I will describe what independently motivates the claim that these hinges do belong in the set of universal hinges. But first I will also describe the problem that arises as Moyal-Sharrock continues to enumerate the universal hinges.

\footnotetext{
${ }^{8}$ Understanding On Certainty p. 148

${ }^{9}$ Understanding On Certainty p. 149

10 See, e.g., Eric Schwitzgebel's “Acting Contrary to Our Professed Beliefs, or the Gulf Between Occurrent Judgment and Dispositional Belief".

11 Understanding On Certainty p. 149
} 


\section{§2. The Bounds of Sense Are Broader Than Moyal-Sharrock Contends}

Moyal-Sharrock goes on to tell us that to the above set of universal hinges we can add "diverse other universal hinges", besides the ones corresponding to Moore's truisms, belong to the set of universal hinges, again without argument. ${ }^{12}$ Some of the hinges she suggests that we can add to the set are "Humans cannot turn into birds or birds into humans" and "A human baby cannot look after itself", "Human beings are not made of glass" and, in another chapter, "I have a brain".13. I agree with her that Wittgenstein seems to treat these claims as hinges, but disagree with the claim that he would have thought of them as universal hinges.

The demonstration that these extra hinges cannot be universal is fairly simple. Consider "I have a brain". The existence of brains must have been an early scientific discovery; thus, some sort of practice of empirical enquiry must have been in place before any human could have truly had the hinge "I have a brain". But on Moyal-Sharrock's account, first discoveries are treated as empirical propositions, and then after repeated exposure, they are fused into our bedrocks and become hinges instead ..$^{14}$

Thus, not only did the agents discovering brains need to have some sort of fairly complex practice in place before discovering brains, but they also needed to, for some time, treat "I have a brain" as an empirical proposition before having it as a hinge. But MoyalSharrock seems to be committed to saying that the humans who lived prior to the discovery of brains were beyond the bounds of human sense - beyond the minimal form of life that we all participate in. This can't be right.

When Moyal-Sharrock claims that "I have a brain" is a universal hinge, she does not actually argue for it, but she does cite On Certainty $§ 159$, and so it would be worthwhile to examine it and see if it provides grounds for taking "I have a brain" to be a universal hinge. The passage reads: "As children, we learn facts; e.g., that every human has a brain, and we take them on trust". So it seems like Moyal-Sharrock has mistaken Wittgenstein to be using

\footnotetext{
12 Understanding On Certainty p. 150

${ }^{13}$ Understanding On Certainty p. 10

${ }^{14}$ Understanding On Certainty p. 155
} 
"we" to refer to "all humans" or "everyone within the bounds of sense" rather than something more cautious, like "my contemporaries".

It seems more charitable to think that Wittgenstein is making the more cautious claim here. This helps us resist attributing to him the view that before children learn that they have brains, they actually stand outside the bounds of sense. Even if there is some stage of development before which children stand inside the bounds of sense, surely learning about their brains is not what makes the difference.

In general, this precise problem and alternative more charitable explanation arises for most of the implausible universal hinges that Moyal-Sharrock considers. That is, for most of the hinges that she counts as universal, it seems as though she did this on the basis of Wittgenstein saying that such-and-such a hinge was a hinge for him and for his readers. While claims of this sort are compatible with the thesis that Wittgenstein thought that they concerned universal hinges, they are also compatible with the thesis that Wittgenstein thought that they concerned local hinges, and this is the more plausible reading.

Now I'll run through this argument a few more times through citing specific universal hinges that Moyal-Sharrock attributes to Wittgenstein alongside the passages from On Certainty that allegedly provide support for this interpretation. This is a useful passage in which Moyal-Sharrock identifies some of her universal hinges and their sources in the text all at once, which I'll use to structure my discussion:

'The earth exists', 'There are physical objects', 'Things don't systematically disappear when we're not looking', 'If someone's head is cut off, the person will be dead and not live again', 'Trees do not gradually change into men and men into trees', 'I have a brain', 'I am a human being', 'I have forbears' (OC 209, 35-6, [134], 274, 513,159, 4, 234, from Understanding On Certainty p. X). ${ }^{15}$

Now I'll demonstrate that the text better supports interpreting most of these hinges as local hinges rather than universal hinges.

Let's begin first with "The earth exists" as a universal hinge, which Moyal-Sharrock

${ }_{15}$ Understanding On Certainty p. 104 cites section 234 in this list rather than 134, which is the correct citation. Thank you to an anonymous referee for raising this to my attention. 
bases on On Certainty §209. Claiming that "The earth exists" is a universal hinge seems to render the bounds of sense overly narrow: "after all, what are we to say of the perfectly sensible people that existed and talked about this earth before calling it the earth, or using any particular concept to refer to the earth?

On Certainty $\$ 209$ reads: "The existence of the earth is rather part of the whole picture which forms the starting-point of belief for me." (emphasis mine) Moyal Sharrock's interpretation seems to miss that in this passage Wittgenstein says "for me". What he says is also perfectly compatible that "The Earth exists is a local hinge, and moreover that view does not face the problem of narrowness that I have raised.

Next we can move on to "I have forebears", which Moyal Sharrock bases on On Certainty §234. Again, to insist that the agent who for whom such a proposition does not go without saying stands outside the bounds of sense seems overly restrictive. On Certainty $\S 234$ reads:

"I believe that I have forebears, and that every human being has them. I believe that there are various cities, and, quite generally, in the main facts of geography and history. I believe that the earth is a body on whose surface we move and that it no more suddenly disappears or the like than any other solid body: this table, this house, this tree, etc. If I wanted to doubt the existence of the earth long before my birth, I should have to doubt all sorts of things that stand fast for me.

Moyal-Sharrock claims, on the basis of this passage, that "I believe that I have forebears" is a universal hinge. But again, in this passage Wittgenstein is making a claim about what he believes and what the people around him believe rather than a claim about the bounds of sense in general. This is compatible with reading him as making a claim about a local hinge rather than a universal hinge. And reading him as making a claim about local hinges is more charitable, on account of the fact that it doesn't attribute an overly narrow account of the bounds of sense to him.

Next we can move on to "If someone's head is cut off he is dead and will never live again”, which Moyal-Sharrock bases on On Certainty §274. On Certainty §274 reads: 
One such is that if someone's arm is cut off it will not grow again. Another, if someone's head is cut off he is dead and will never live again. Experience can be said to teach us these propositions. However, it does not teach us them in isolation: rather, it teaches us a host of interdependent propositions. If they were isolated I might perhaps doubt them, for I have no experience relating to them.

In this passage, Wittgenstein says that this is a hinge had by "us". But the "us" here could refer to his contemporaries rather than any agent that stands within the bounds of sense. And it seems implausible to suggest that an agent who believes that one can recover from losing their had stands outside the bounds of sense. Yes, such an agent is wrong, but having a false belief is not sufficient for standing outside the bounds of sense.

Moyal-Sharrock's underlying method of identifying universal hinges seems to be descriptive rather than explanatory or predictive. What I mean by a descriptive method of identifying hinges is the following method: imagine that we could represent each human's bedrock as a set $H$ containing hinges $\left\{h_{1}, h_{2}, \ldots\right\}$ for every hinge the human has in their bedrock. We might, then, be tempted to conclude that given the set of hinges for every past, future, and present human, we could look at the intersection of all the sets and then conclude that we had discovered the set of universal hinges- after all, the intersection of all these sets is the set of hinges that all humans share.

The problem with this method is that it will identify too many hinges - not only will it identify the genuinely universal hinges, it will simply also identify the local hinges that all cultures, as a matter of coincidence, happen to share. This is something like the mistake a psychologist makes when they observe a cultural universal and then conclude that the universal has emerged as a result of some characteristic of human nature. Such a conclusion might be true, but it might also be the case that similar groups of people, facing similar challenges, have in many cases developed similar ways of addressing those challenges.

This, I think, explains why Moyal-Sharrock thinks it is plausible to say that hinges like "Humans cannot turn into birds" are universal. She believes that knowing about birds and their properties is part of the human form of life - perhaps because of the assumption 
that all human cultures have come into contact with birds, and noticed that humans cannot turn into birds. Fair enough: they all probably have come into contact with birds, and made such a realization. But that does not entail that that does not entail that a failure to come into contact with birds and think about the relationship between birds and humans consigns someone to stand without the bounds of sense.

It is a mistake to think that accidents like this should have any bearing on our understanding of what it is to stand within the human bounds of sense - we ought to be able to account for possible cases like the possible group of people that has never seen birds, and to correctly conclude they still stand within the human bounds of sense. Our method of identifying universal hinges should, instead, be explanatory or predictive: we should first understand what universal hinges are, and then use that understanding to generate a list of the universal hinges. ${ }^{16}$

\section{§3. A Positive, Explanatory, Predictive Account of Universal Hinges}

What is it to be rational? How should we really answer the question, "Which hinges constitute the bounds of sense?" These questions are too large to persuasively answer, in a general sense, in one paper. What I will argue is that Wittgenstein's answer to this question might rule out the hinges that I have contended are local rather than universal. Getting clear on Wittgenstein's contribution to this debate will not settle it, but it will be a contribution that helps move the conversation forward.

Wittgenstein's answer to such a question is probably that what unifies all human forms of life is having the ability to participate in language games; specifically, having the ability to participate in relatively complex language games - typical human speech rather than the slab fetching discussed early in the Philosophical Investigations. Then we need to ask what sort of animal certainties an agent must have to have the ability to participate in

\footnotetext{
16 In Understanding On Certainty, after introducing universal hinges, Moyal-Sharrock says that she will address the objection that they are not genuinely universally shared (p. 103). But she does not consider cases involving a simple lack of capacity, as I do here. Instead, she considers cases where members of certain cultures seem to have hinges or beliefs that are the negations of her universal hinges (pp. 175-76). But my category of counterexample is different in kind from this sort of counterexample. My counterexamples do not concern people who believe that we can turn into birds, but instead just people who don't think anything at all about birds; similarly, my counterexamples do not concern people that think we have sawdust instead of brains in our skulls, but instead just people who lack any beliefs about brains (and so on).
} 
language games - those certainties are the universal hinges. Agents who lack those certainties are the ones who are actually beyond the human bounds of sense. Thinking of things in these terms, I contend, rules out Moyal-Sharrock's implausible universal hinges (like "I have a brain") and rules in her plausible universal hinges (like "The world exists" or "There are other minds").

First, consider "the world exists". Given that gaining the ability to participate in language games requires extensive training, any agent that has learned to participate in language games will have to be relatively skilful at interacting with the world - any agent with such a skillset must, in their lives, display that "the world exists" is in their bedrock. Similarly, it looks like several of Moore' truisms are the sorts of things that must be hinges in someone's bedrock in order for them to acquire the ability to participate in complex language games. For example, Moore says that he knows with certainty that he has perceived other humans, and we do need to interact with other humans while we are young in order to acquire language.

Now consider some of Moyal-Sharrock's problematic hinges- "I have a brain", for example. Do agents need to have that hinge in their bedrock in order to have the ability to participate in complex language games? Obviously not: there are a lot of language games that don't involve the relatively complex concept "brain", and it's complex enough that it should be clear that having a hinge concerning brains is not something that could form the

foundation of a basic human ability. Similarly the case for "Men don't turn into birds"- while this is true, and obvious for any member of a culture that is exposed to birds, it is just as obvious that there is no conceptual link between having the ability to participate in complex language games in general and having a hinge concerning the question of whether men can turn into birds.

\section{§4. Hinges and Foundationalism}

I think my account of the universal hinges, while it simply enjoys independent motivation as I argued above, should also be adopted because it would help MoyalSharrock respond to an objection that Michael Williams and Annalisa Coliva have raised to the contention that hinges can be used roughly as "basic beliefs" in a foundationalist 
epistemology. First I'll explain this objection, and why Moyal-Sharrock's current response to the objection is unsatisfying. Then I'll explain why my account of the universal hinges allows us to better support the view that hinges can play a role in a foundationalist epistemology.

Michael Williams has articulated this objection in his paper "Why Wittgenstein Isn't a Foundationalist". There are two steps: first, he claims that for the foundationalist, the foundations of our beliefs must be universally shared by all knowers: Williams writes:

"This is certainly the view of traditional foundationalists, for whom genuine foundations are, indeed must be, universal. We might say, this commitment is what makes foundationalism a theory of knowledge."17

Next, he claims that Wittgenstein's hinges are clearly not universally shared by knowers, and therefore obviously not fit to play the role of the foundations of our knowledge:

This objection brings us back to universality, for it underestimates the degree of heterogeneity that Wittgenstein finds in 'basic' certainties. Some certainties are perceptual judgments about objects in our surroundings ('Here is one hand'). Others are general propositions, belonging to the scaffolding of our Moorean common sense ('The Earth has existed for many years past'). Still others are presuppositions of quite specialized, thus potentially culturally specific, forms of inquiry (history, geology, physics). Thus while some certainties may be universal, others need not be, and some manifestly aren't. ${ }^{18}$

Thus, according to Williams, hinges vary between people and times and cultures, which prevents them from being universal, which prevents them from play a role in a foundationalist epistemology.

Annalisa Coliva has expressed the same point, for instance in her 2012 reply to Moyal-Sharrock and other commentators:

As to (i), of course some of Wittgenstein's hinges are universal, but

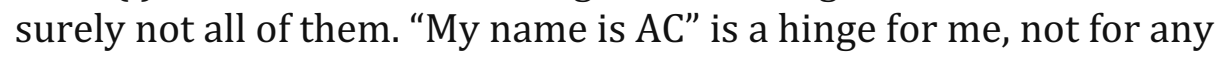

17 "Why Wittgenstein Isn't a Foundationalist" p. 50

18 "Why Wittgenstein Isn't a Foundationalist" p. 54 
other person, although it is to be expected that each person will have his own similar hinge-i.e. "My name is NN". ${ }^{19}$

Like Williams, Coliva believes that hinges will vary too much between people to serve as the foundations of our beliefs.

At this point in the paper, it should be clear both what Moyal-Sharrock's answer to this objection is, and how my view of universal hinges improves her answer. MoyalSharrock's answer to this objection is to say that there is a special class of hinges such that they are shared by everyone within the bounds of sense - the aptly named "universal hinges" - and that these hinges are suitable to play a role in a foundationalist epistemology. ${ }^{20}$

But the problem is that Moyal-Sharrock's universal hinges are still relatively heterogeneous, and include hinges that are not shared by everyone within the bounds of sense, such as "I have a brain". Perhaps this is why commenters like Williams and Coliva have continued to press their point. Given the way that Moyal-Sharrock develops the concept of a universal hinge, it really is non-obvious that the concept of universal hinges does anything to address this objection on the grounds of universalizability.

However, adopting my account of the universal hinges, according to which the universal hinges are the ones that are necessary to participate in language games, does give Moyal-Sharrock the resources to address the problem raised by Williams and Coliva. Once our notion of the universal hinges is sparser than Moyal-Sharrock's original notion, the universal hinges stop seeming so heterogeneous, and start seeming like they might be the genuinely universal foundations of our knowledge. ${ }^{21}$

\footnotetext{
19 “Replies" p. 85

${ }^{20}$ She has made this reply in "On Coliva's Judgmental Hinges" p. 24

21 Moyal-Sharrock has also put the universal hinges to the theoretical use of explaining the sense in which Wittgenstein is not a relativist (see Moyal-Sharrock 2017). She argues that the universal hinges provide a cross-cultural standard for a shared human form of life. Like Moyal-Sharrock, I think that this is an urgent and important theoretical use for the universal hinges, but I argue that the category of universal hinges needs to be refined before we put it to work like this.
} 


\section{§5. Universal Hinges, Pritchard's über hinge, and Coliva's extended rationality}

Finally, I'd like to consider my account of the universal hinges in relation to two similar concepts: Duncan Pritchard's "über hinge", and Annalisa Coliva's "extended rationality". In Epistemic Angst, Pritchard notes that at first pass, the category of hinges seems rather heterogeneous:

“... Wittgenstein writes, for example, about hinges concerning the fact that one has never been to the moon (e.g., OC, §106) or that one's name is such and such (e.g., OC §425). But in each case the 'hinge' in question seems very relative to the particular circumstances of the person in question: what age they live in, who they are, where they were raised, and so on." (Pritchard 2016 p. 95)

Pritchard then argues that this heterogeneity is actually delusive, and that all the hinges are derived from one hinge which is shared across times and cultures. He calls this hinge the "über hinge", and says that the über hinge commitment is the "entirely general hinge commitment that one is not radically and fundamentally mistaken in one's beliefs". (Pritchard 2016 p. 96) According to Pritchard, at various times and places, this hinge causes, or does not cause, subjects to have hinges like "No one has ever been to the moon".

We can see Pritchard's account as offering a competing answer to the question of this paper, "How should we characterize the universal hinges?", although I should emphasize that Pritchard does not explicitly make that suggestion here himself. According to this proposal, we should say that there is just one universal hinge, the über-hinge, rather than identifying the universal hinges with the range of hinges that enable participation in language games. I think that the proposal that the universal hinges just are the über-hinge fails as an interpretation of Wittgenstein in On Certainty because of Wittgenstein's view on the relationship between doubt and belief. Wittgenstein wrote: "The child learns by believing the adult. Doubt comes after belief." (§160) I read this passage as not making a purely developmental claim, but also more broadly making a claim about which capacity is more fundamental than the other - namely, that belief is more fundamental than doubt. And although this passage is about belief and doubt rather than hinges, I think it's reasonable to infer from this passage that Wittgenstein would say that simple declarative 
hinges, like "men cannot turn into birds", come before more complex hinges concerning doubts, like "I am not currently radically deceived".

But on Pritchard-inspired proposal that I am considering here, the bounds of sense are constituted by the über -hinge, and the über-hinge causes us to have various particular hinges, like that (e.g.) people cannot turn into birds or that the earth exists. (And I note again that this is a proposal that I am entertaining in this section, rather than a proposal that Pritchard has made explicitly.) Therefore, through suggesting that the hinge related to entertaining and rejecting doubts is more fundamental or primitive than hinges related to forming beliefs or taking other actions related to birds and the earth, the Pritchard-inspired proposal under consideration seems get things backwards from the Wittgensteinian perspective articulated in $\S 160$. We should prefer accounts of the universal hinges that cohere better with that perspective, and my proposal in this paper is one such account. It also seems like children who have achieved the capacity to believe but not yet achieved the capacity to entertain and reject sceptical scenarios stand within the bounds of sense, contrary to the prediction that the the über-hinge is what constitutes the bounds of sense.

Now we can move on to the relationship between my view and Coliva's extended rationality view. In Extended Rationality, Coliva presents a view of rationality in general and applies it to skeptical problems. Coliva argues that certain assumptions, like "there is an external world", are partly constitutive of rationality, and shared by the skeptic and nonskeptic alike (pp. 128-129). The account she presents is Wittgenteinian in spirit, although not intended to be a direct reading of an account of rationality that Wittgenstein endorsed in On Certainty (throughout the course of her book, Coliva develops a variety of interesting views and families of views that respond to the problem of skepticism).

It's worth remarking, however, on the similarities between her account of rationality and the account of rationality that I have generated through analyzing textual evidence concerning the category of the universal hinges in On Certainty. Although Coliva does not fully specify the list of assumptions that she takes to be constitutive of rationality, the list includes assumptions like "There is an external world", "Our sense organs are mostly working reliably", and "One is not the victim of a lucid and sustained dream", and so 
her list overlaps with the list of universal hinges that I have developed over the course of this paper. This overlap speaks in favour of the idea that Coliva and Wittgenstein converged upon the same concept of rationality, rather than one which was just similar in spirit. While her explanatory goal is finding the best concept of rationality for her purposes in Extended Rationality, rather than finding the concept of rationality outlined by the universal hinges, it seems to me that both those goals take us to the same place.

That being said, I also think that it is a virtue of Wittgenstein's account, and MoyalSharrock's account, to explicitly preserve the category of the local hinges. The local hinges are things that we use in just the same way that we use the universal hinges, but they vary across time and place in dramatic ways - notoriously, Wittgenstein had a hinge concerning the impossibility of travel to the moon, and we do not (\$108). Coliva's account of rationality does not seem sensitive to these variations in how humans in different times and places engage in belief formation, which focuses on addressing traditional skeptical challenges about the external world rather than the more day-to-day belief formations and actions that are guided by the local hinges. ${ }^{22}$

\section{$\S 5$. Conclusion}

I have argued that many hinges Moyal-Sharrock takes to be universal are actually local. This problem motivated me to present a new general principle that explains what universal hinges are: they are the animal certainties that constitute (or at least, are necessary for having) the ability to participate in language games. This principle generates a suitably broad account of the bounds of sense and is compatible with Wittgenstein's various remarks about hinges.

\footnotetext{
${ }^{22}$ Here is a further note about the heterogeneity of hinges. Ashton (2019) points out that factors like race and gender, as well as e.g. locality, the narrowest factor that I consider here, can contribute to the justification that a subject has, and probably also to the hinges that a subject has. This seems like an important point to keep in mind for theorists developing their own hinge epistemologies today and going forwards. I don't include these factors in this discussion because (in this paper) I am one of the writers on hinges focusing on what Ashton calls "faithfulness": "whether or not the account [of hinges] faithfully reconstructs the text of On Certainty and accurately represents Wittgenstein's intentions." (p. 155) To my knowledge, his hinges sometimes seem local, and sometimes seem personal, but don't seem to concern social categories, which would stand in between those two levels of specificity.
} 
I will close with considering a couple of potential objections. First, my notion of what it is to be in the human bounds of sense is in fact so broad that it will include, say, hypothetical intelligent Martians who know nothing of what Moyal-Sharrock included in the human form of life - for instance, the hinge proposition that humans cannot turn into birds - but who are capable of participating in complex language games of their own. And perhaps such a notion of the human form of life seems too broad.

This line of thought is mistaken. Moyal-Sharrock's account of the human form of life focuses too much on biological trivia ("humans cannot turn into birds, "human babies need to be cared for"). But what seems to be genuinely important about the bounds of sense is something other than a hinge concerning biological trivia. From the Wittgensteinian perspective, in any case, what matters are things like rationality, complexity of thought, and the ability to communicate. Agents with these features and abilities stand within the bounds of sense, and those who lack them stand without those bounds. If there turn out to be Martians with language games of their own, then surely they belong in the bounds of sense, just as much as a human that can participate in language games but that doesn't know anything about the brain.

Secondly, I will consider an objection about whether my interpretation of Wittgenstein violates his constraints on philosophizing. I have argued, so far, for two independent claims. First, I have argued that Moyal-Sharrock's list of universal hinges simply seems too long, and to draw the bounds of human sense too narrowly. Second, I have argued that we can use an explanatory, predictive account of hinges to understand Wittgenstein's actual account of the universal hinges. This explanatory and predictive account is motivated by Wittgenstein's various remarks about hinges. Given Wittgenstein's frequent exhortations to describe rather than explain: for instance, "I want to say here that it can never be our job to reduce anything to anything, or to explain anything. Philosophy really is 'purely descriptive'." (1958 p. 18), one might wonder whether I have offered an unWittgensteinian explication of Wittgenstein's views. However, it is not my claim that he endorsed this explanatory account explicitly. Thus, I am not claiming that Wittgenstein set out to violate his dictum against explaining. Instead, I have argued that - perhaps 
surprisingly - doing some explaining can help us understand Wittgenstein's concept of the universal hinges.

More broadly, I think we should expect different styles of philosophizing to be suitable to different contexts, and that in some contexts more or less explanation might be called for. As my work in this paper shows us, this particular context is one in which some description and some explanation can, together, help us achieve our goal of understanding the universal hinges.

\section{References}

Ashton, N. (2019). "The Case for a Feminist Hinge Epistemology”. Wittgenstein-Studien. 10 (1):153-163

Coliva, A. (2013). “Replies”. Philosophia 41:81-96

Coliva, A. (2015). Extended Rationality: A Hinge Epistemology. Palgrave-Macmillan.

Moyal-Sharrock, D. (2004). Understanding Wittgenstein's On Certainty. Basingstoke: Palgrave Macmillan.

Moyal-Sharrock, D. (2013) “On Coliva’s Judgmental Hinges” Philosophia 41:13-25.

Moyal-Sharrock, D., \& Brenner, W. H. (Eds.). (2005). Readings of Wittgenstein's On Certainty. Basingstoke: Palgrave Macmillan.

Moyal-Sharrock, D. (2017). "Fighting Relativism: Wittgenstein and Kuhn". In Katharina Neges, Josef Mitterer, Sebastian Kletzl \& Christian Kanzian (eds.), Realism - Relativism Constructivism: Proceedings of the 38th International Wittgenstein Symposium in Kirchberg. De Gruyter. pp. 215-232.

Pritchard, D. (2016). Epistemic Angst: Radical Skepticism and the Groundlessness of Our Believing. Princeton University Press.

Schwitzgebel, E. (2010). "Acting Contrary to Our Professed Beliefs, or The Gulf Between Occurrent Judgment and Dispositional Belief". Pacific Philosophical Quarterly, 91, 531-553.

Williams, M. (2005) “Why Wittgenstein Isn't a Foundationalist” in Moyal-Sharrock \& Brenner (2005), 47-58.

Wittgentein, L. (1958). The Blue and Brown Books. Harper and Row.

Wittgenstein, L. (1969). On Certainty. Oxford: Blackwell. 